\title{
MicroRNA-Related Polymorphisms in PI3K/Akt/mTOR Pathway Genes Are Predictive of Limited-Disease Small Cell Lung Cancer Treatment Outcomes
}

\author{
Wei Jiang, ${ }^{1,2}$ Wenjue Zhang, ${ }_{1}^{1}$ Lihong Wu, ${ }^{1}$ Lipin Liu, ${ }^{1}$ Yu Men, ${ }^{1}$ Jingbo Wang, \\ Jun Liang, ${ }^{1}$ Zhouguang Hui, ${ }^{1}$ Zongmei Zhou, ${ }^{1}$ Nan Bi, ${ }^{1}$ and Luhua Wang ${ }^{1}$ \\ ${ }^{1}$ Department of Radiation Oncology, National Cancer Center/Cancer Hospital, Chinese Academy of Medical Sciences and \\ Peking Union Medical College, Beijing 100021, China \\ ${ }^{2}$ Department of Radiation Oncology, Cancer Hospital, Chinese Academy of Medical Sciences, Shenzhen Center, \\ Shenzhen 100021, China
}

Correspondence should be addressed to Nan Bi; binan_email@163.com and Luhua Wang; wlhwq@yahoo.com

Received 26 July 2016; Revised 30 November 2016; Accepted 15 December 2016; Published 9 February 2017

Academic Editor: Franco M. Buonaguro

Copyright (c) 2017 Wei Jiang et al. This is an open access article distributed under the Creative Commons Attribution License, which permits unrestricted use, distribution, and reproduction in any medium, provided the original work is properly cited.

\begin{abstract}
The phosphoinositide-3 kinase (PI3K)/Akt/mammalian target of rapamycin (mTOR) signaling pathway plays an important role in cancer progression and treatment, including that of small cell lung cancer (SCLC), a disease with traditionally poor prognosis. Given the regulatory role of microRNA (miRNA) in gene expression, we examined the association of single nucleotide polymorphisms (SNPs) at miRNA-binding sites of genes in the mTOR pathway with the prognosis of patients with limited-disease SCLC. A retrospective study was conducted of 146 patients with limited-disease SCLC treated with chemoradiotherapy. Nine SNPs of six mTOR pathway genes were genotyped using blood samples. Cox proportional hazard regression modeling and recursive partitioning analysis were performed to identify SNPs significantly associated with overall survival. Three SNPs, MTOR: rs2536 (T>C), PIK3R1: rs3756668 (A>G), and PIK3R1: rs12755 (A>C), were associated with longer overall survival. Recursive partitioning analysis based on unfavorable genotype combinations of the rs 2536 and rs 3756668 SNPs classified patients into three risk subgroups and was internally validated with 1000 bootstrap samples. These findings suggest that miRNA-related polymorphisms in the $\mathrm{PI} 3 \mathrm{~K} / \mathrm{Akt} / \mathrm{mTOR}$ pathway may be valuable biomarkers to complement clinicopathological variables in predicting prognosis of limited-disease SCLC and to facilitate selection of patients likely to benefit from chemoradiotherapy.
\end{abstract}

\section{Introduction}

Small cell lung cancer (SCLC), which accounts for approximately $13 \%$ of lung cancer cases [1], is a common neuroendocrine malignancy characterized by aggressive growth and early metastasis. Chemotherapy with agents of etoposide or topoisomerase 1 inhibitors (irinotecan or topotecan), along with cisplatin and radiotherapy, is the recommended treatment for SCLC with limited disease (LD SCLC) [2, 3]. Unfortunately, there is only a $15 \%$ to $20 \%$ cure rate and most cases recur rapidly, leading to a very poor overall prognosis [4]. Genetic variations may play an important role in treatment sensitivity; therefore, identification of novel genetic predictors could be helpful for individualized treatment options.

The phosphoinositide-3 kinase (PI3K)/Akt/mechanistic target of rapamycin (mTOR) signaling pathway has been a hot therapeutic target for SCLC in recent research [5]. This pathway is activated in various cancer types, including SCLC [6], and is associated with radiation and chemotherapy resistance [7]. Genetic variations in this pathway are reported to modulate clinical outcomes in patients with esophageal cancer and non-small-cell lung cancer who have undergone chemoradiotherapy $[8,9]$. MicroRNAs (miRNAs), a class of small, noncoding RNAs, are key regulators of gene expression in many biological processes [10], including the mTOR 
pathway [11]. Single nucleotide polymorphisms (SNPs) in miRNA-binding sites may affect the regulatory effect of miRNAs on oncogenes and tumor suppressor genes. However, little published research has considered the impact of miRNArelated genetic polymorphisms in the $\mathrm{PI} 3 \mathrm{~K} / \mathrm{Akt} / \mathrm{mTOR}$ pathway and their relationship to SCLC outcome. We therefore analyzed the effect of these genetic variations on the prognosis of patients with LD SCLC receiving curative chemoradiotherapy and selected valuable biomarkers for decision making.

\section{Materials and Methods}

2.1. Ethics Statement. This investigation was conducted in accordance with the ethical standards outlined in the Declaration of Helsinki and national and international guidelines. Our institutional review board (Cancer Hospital, Chinese Academy of Medical Sciences) approved this retrospective study, and informed consent was waived.

2.2. Study Population. Patients in this study were retrospectively recruited from the Cancer Hospital, Chinese Academy of Medical Sciences, between January 2007 and June 2014. All SCLC were histologically confirmed, staged as LD SCLC based on the International Association for the Study of Lung Cancer (IASLC) classification [12], and initially treated with curative-intent platin-based chemotherapy combined with intensity-modulated radiation therapy (IMRT). Patients who had any other malignancy within five years of enrollment were excluded to avoid a potential confounding effect. Prophylactic cranial irradiation (PCI) was offered to patients who had achieved a complete remission (CR) or partial response (PR), depending on the treating physician's discretion and patient's preference. Clinical information was collected from medical records. Long-term archived serum samples from recruited patients were analyzed [13].

2.3. SNP Selection and Genotyping. Candidate miRNArelated SNPs of $\mathrm{PI} 3 \mathrm{~K} / \mathrm{Akt} / \mathrm{mTOR}$ pathway genes were selected in two ways: SNPs located at the $3^{\prime}$-UTR of miRNA target genes with a minor allele frequency greater than 0.1 in the Chinese Han population in the PolymiRTS Database 3.0 (http://compbio.uthsc.edu/miRSNP/) and Ensemble Asia database (release 79, http://asia.ensembl.org/in-dex.html? redirect=no) as well as those previously reported in the literature to be associated with cancer were included. Tagging SNPs in linkage disequilibrium were identified using HaploReg version 3 (http://www.broadinstitute.org/mammals/ haploreg/haploreg_v3.php) with a cut-off value of $r^{2}>0.8$.

Genomic DNA was extracted using a TIANamp Blood DNA Kit (TIANGEN Biotech, Beijing, China). Genotyping was conducted using the MALDI-TOF mass spectrometrybased iPLEX Gold assay on the Sequenom MassARRAY Platform (San Diego, CA, USA) and was analyzed using MassARRAY TyperAnalyzer v4.0 software (Sequenom). SNPs with a more than $95 \%$ success rate and samples with a call rate exceeding $96 \%$ were included. Ultimately, a total of nine tagSNPs in six genes were selected.
2.4. Statistical Analysis. Response rates were evaluated according to RECIST 1.0 criteria. The objective response rate (ORR) included CR and PR to treatment. The association of genetic features with ORR was estimated using the Pearson $\chi^{2}$ test with odds ratios (ORs) and 95\% confidence interval (CI). Overall survival (OS) was calculated from pathologic diagnosis to the date of death or last follow-up. The KaplanMeier method and log-rank test were used to assess survival for each genotype and clinical characteristics. Hazard ratios (HRs) and 95\% CI were estimated by Cox proportional hazards regression models. The adjustment factors included age, gender, smoking history, Karnofsky performance score (KPS), and Charlson comorbidity index (CCI). Genotype analyses were conducted in three genetic models (dominant, recessive, and additive) for each SNP and the model with the smallest $P$ value was used. Models with rare genotypes $(<5 \%$ of patients) were excluded. Recursive partitioning analysis (RPA) was conducted to evaluate the cumulative effects of the genetic variants in the pathway. Concordance probability estimate (CPE) was used to assess the predictive ability of RPA classification [14] from 1000 bootstrap samples.

A two-sided $P$ value $<0.05$ was considered significant for all statistical analyses. Multiple comparison was performed by Benjamini-Hochberg False Discovery Rate (FDR) correction based on tests for 3 models with a $q$-value of 0.05 [15]. Statistical power of the RPA classification was calculated with an $\alpha$ of 0.1 . All statistical analyses were carried out using IBM SPSS Statistics 21.0 software (IBM Corp., Armonk, NY) and R version 3.2.3 (http://www.r-project.org.com/).

\section{Results}

3.1. Clinical Characteristics. The clinical characteristics of 146 patients with LD SCLC recruited to this study are shown in Table 1. Patients' median age at diagnosis was 56.8 years (range 29-80 years). Of these patients, 91.8\% were diagnosed as stage III. Chemotherapy was delivered based on an EP (etoposide + cisplatin) or EC (etoposide + carboplatin) regimen, usually for 2-4 cycles; concurrent treatment was given to $54.8 \%$ patients with no difference in overall survival compared to those receiving sequential treatment. A total of 37 patients achieved CR and 84 had a PR. PCI was administered to $43.2 \%$ patients, $90.5 \%$ of whom had a CR or PR in the primary lesion. The overall median survival time (MST) and 5-year OS rate were 35.1 months and 38.9\%, respectively, at a median follow-up time of 42.2 months. Age, KPS, CCI, and PCI were prognostic factors for OS $(P<0.05$, log-rank test).

3.2. Associations of Individual SNPs with Outcome. The survival analysis by genotype for each SNP is shown in Table 2. Two SNPs were significantly associated with OS (MTOR: rs2536 and PIK3R1: rs3756668), rising to three (PIK3R1: rs12755) after adjustment for age, gender, KPS, smoking history, and CCI. Patients carrying heterozygous TC of MTOR: rs2536 (T $>C$ ) had a significantly increased risk of reduced OS compared to those with TT genotype (adjusted HR = 1.948, 95\% CI: 1.090-3.482). In addition, variant homozygous genotypes of rs12755 $(A>C)$ and $\operatorname{rs3756668}(A>G)$ in PI3K 
TABLE 1: Clinical characteristics of 146 patients with limited diseasesmall cell lung cancer.

\begin{tabular}{|c|c|c|c|}
\hline Variables & $N(\%)$ & $5 y O S$ & $P$ \\
\hline \multicolumn{4}{|l|}{ Gender } \\
\hline Male & $104(71.2 \%)$ & $33.0 \%$ & \multirow{2}{*}{0.065} \\
\hline Female & $42(28.8 \%)$ & $47.2 \%$ & \\
\hline \multicolumn{4}{|l|}{ Age } \\
\hline$\leq 60$ & $95(65.1 \%)$ & $43.0 \%$ & \multirow{2}{*}{0.015} \\
\hline$>60$ & $51(34.9 \%)$ & $30.8 \%$ & \\
\hline \multicolumn{4}{|l|}{ KPS } \\
\hline$\geq 90$ & $61(41.8 \%)$ & $47.5 \%$ & \multirow{2}{*}{0.009} \\
\hline$<90$ & $85(58.2 \%)$ & $33.1 \%$ & \\
\hline \multicolumn{4}{|l|}{ Location } \\
\hline Left lobe & $69(47.3 \%)$ & $39.3 \%$ & \multirow{2}{*}{0.987} \\
\hline Right lobe & $77(52.7 \%)$ & $37.8 \%$ & \\
\hline \multicolumn{4}{|l|}{ Smoking } \\
\hline Yes & $96(65.8 \%)$ & $35.7 \%$ & \multirow{2}{*}{0.088} \\
\hline No & $50(34.2 \%)$ & $40.1 \%$ & \\
\hline \multicolumn{4}{|c|}{ Charlson comorbidity index } \\
\hline$\leq 3$ & $130(89.0 \%)$ & $41.7 \%$ & \multirow{3}{*}{0.030} \\
\hline $4-5$ & $13(8.9 \%)$ & $19.5 \%$ & \\
\hline $6-7$ & $3(2.1 \%)$ & $0 \%$ & \\
\hline \multicolumn{4}{|l|}{ Weight loss } \\
\hline With & $27(18.5 \%)$ & $51.3 \%$ & \multirow{2}{*}{0.393} \\
\hline Without & $119(81.5 \%)$ & $35.2 \%$ & \\
\hline \multicolumn{4}{|l|}{ AJCC stage } \\
\hline IA & $1(0.7 \%)$ & $100.0 \%$ & \multirow{6}{*}{0.573} \\
\hline IB & $2(1.4 \%)$ & $0 \%$ & \\
\hline IIA & $5(3.4 \%)$ & NA & \\
\hline IIB & $4(2.7 \%)$ & NA & \\
\hline IIIA & $68(46.6 \%)$ & $41.7 \%$ & \\
\hline IIIB & $66(45.2 \%)$ & $34.9 \%$ & \\
\hline \multicolumn{4}{|c|}{ Treatment modality } \\
\hline Concurrent & $80(54.8 \%)$ & $46.8 \%$ & \multirow{2}{*}{0.401} \\
\hline Sequential & $66(45.2 \%)$ & $31.2 \%$ & \\
\hline \multicolumn{4}{|c|}{ Chemotherapy cycles } \\
\hline$<4$ & $9(6.2 \%)$ & NA & \multirow{3}{*}{0.382} \\
\hline $4-6$ & $126(86.3 \%)$ & $40.2 \%$ & \\
\hline$>6$ & $11(7.5 \%)$ & $38.6 \%$ & \\
\hline \multicolumn{4}{|c|}{ Radiotherapy dose } \\
\hline$<60$ & $46(30.7 \%)$ & $39.3 \%$ & \multirow{2}{*}{0.525} \\
\hline$\geq 60$ & $100(69.3 \%)$ & $39.9 \%$ & \\
\hline \multicolumn{4}{|l|}{ PCI } \\
\hline With & $63(43.2 \%)$ & $62.1 \%$ & \multirow{2}{*}{$1.54 E-4$} \\
\hline Without & $83(56.8 \%)$ & $23.1 \%$ & \\
\hline
\end{tabular}

OS: overall survival; AJCC: American Joint Committee on Cancer; PCI: prophylactic cranial irradiation.

regulatory subunit 1 (alpha) (PIK3R1) were associated with $77.5 \%$ (adjusted HR $=0.225,95 \%$ CI: $0.054-0.931$ ) and $61.2 \%$ (adjusted HR $=0.388,95 \%$ CI: 0.176-0.856) decrease in the risk of death, respectively (Figures $1(\mathrm{a})-1(\mathrm{c})$ ). However, after multiple comparison correction, the associations with OS of these SNPs were not statistically significant.

Of the three prognostic SNPs, rs12755 and rs3756668 were associated with ORR of chemoradiotherapy in patients with LD SCLC. Individuals carrying the rs12755 C allele showed a significantly higher ORR than those with AA genotype (ORs $=0.908,95 \%$ CI: $0.858-0.961, P=0.037)$. For rs3756668, carriers of the $\mathrm{G}$ allele had a higher ORR compared with the AA genotype $(\mathrm{OR}=0.790,95 \% \mathrm{CI}=0.720-0.867, P=0.012)$.

3.3. Associations of Individual SNPs with OS in Stage III Patients. Assessing only the 134 patients with stage III SCLC, the overall MST and 5-year OS rate were 35.1 months and 38.2\%, respectively. MTOR: rs2536 (T>C) and PIK3R1: rs3756668 (A>G) remained significantly associated with survival in stage III patients (Table 3). PIK3R1: rs12755 $(\mathrm{A}>\mathrm{C})$ was a borderline prognostic factor after adjusting for clinical covariates (HR adjusted $=0.242$, 95\% CI: $0.058-$ 1.010). However, after correcting for multiple comparison, the discriminations of these genetic variants for survival were not statistically significant (Table 3). Other polymorphisms in the $\mathrm{PI} 3 \mathrm{~K} / \mathrm{Akt} / \mathrm{mTOR}$ pathway continued not to show significant association with OS.

3.4. Recursive Partitioning Analysis (RPA). To explore the combined effect of unfavorable genotypes, three SNPs significantly associated with OS ( $\mathrm{rs} 2536(\mathrm{~T}>\mathrm{C})$ in an additive model, as well as rs12755 and rs3756668 in recessive models), were included in a recursive partitioning analysis. The RPA model was developed based on PIK3R1: rs12755 (A>C) and PIK3R1: rs3756668 (A>G), and the data set was split into three risk classifications (Table 4). The MST and 5-year OS rate were $48.6,35.1$, and 18.4 months and $47.7 \%, 40.8 \%$, and $17.6 \%$ in the low, intermediate, and high-risk classes, respectively, after adjustment for age, gender, KPS, smoking history, and CCI (Figure 1(d)). The ORs of ORR in the intermediate and high-risk classes were 0.757 (95\% CI: 0.679-0.845) and 0.359 (95\% CI: 0.236-0.546) using the low risk group as a reference. The statistical power of this classification was 0.74 .

3.5. Validation of the RPA Classification Using Bootstrap Analysis. Bootstrap analysis was conducted and confirmed a good performance of the RPA predictive model based on $\mathrm{PI} 3 \mathrm{~K} / \mathrm{Akt} / \mathrm{mTOR}$ pathway SNPs in survival discrimination by 1000 resampling internal validation datasets (Table 4 ). The CPE of the RPA classification was 0.69 from 1000 bootstrap samples, supporting the satisfactory predictive efficacy.

\section{Discussion}

SCLC is second only to melanoma in terms of malignancies with a high degree of genomic alternations [16, 17]. As one of the most promising therapeutic targets in SCLC, the PI3K/Akt/mTOR pathway was shown to have a high prevalence of genetic alternations in a comprehensive genomic analysis of SCLC in Asian populations [18]. The $\mathrm{PI} 3 \mathrm{~K} / \mathrm{Akt} / \mathrm{mTOR}$ pathway plays a critical role in cancer progression by regulating cell growth, proliferation, and survival [19]. This pathway was also reported to be involved in 


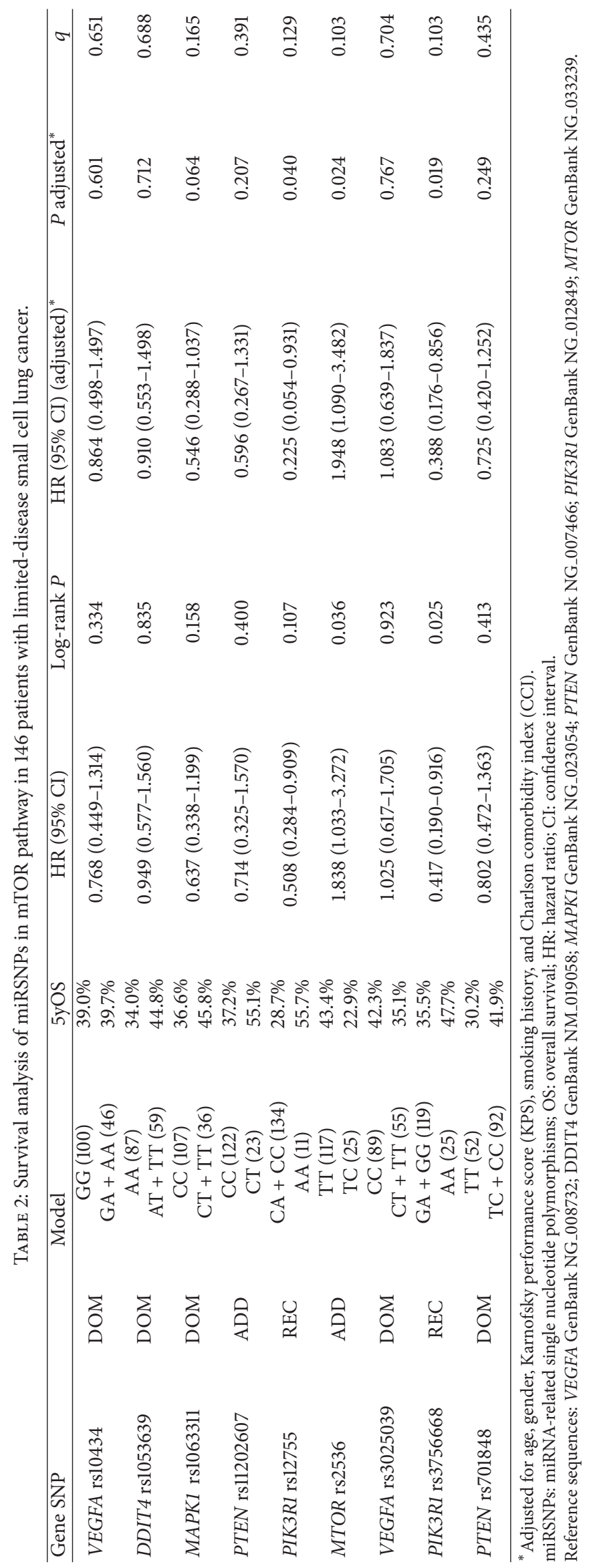




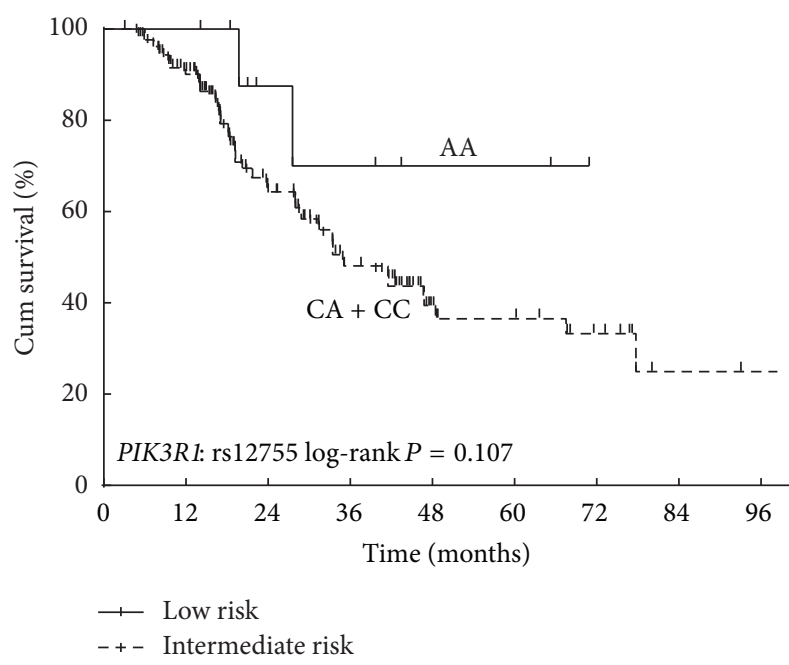

(a)

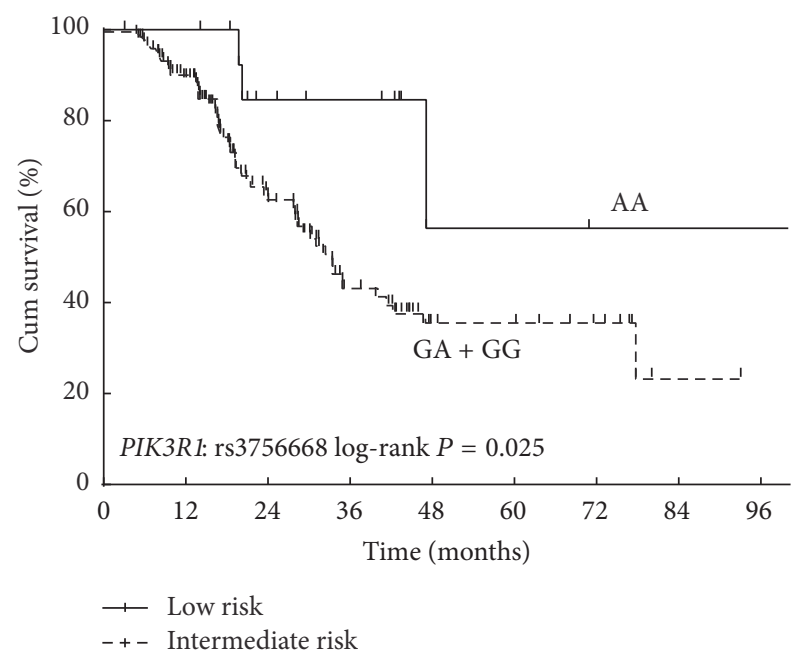

(c)

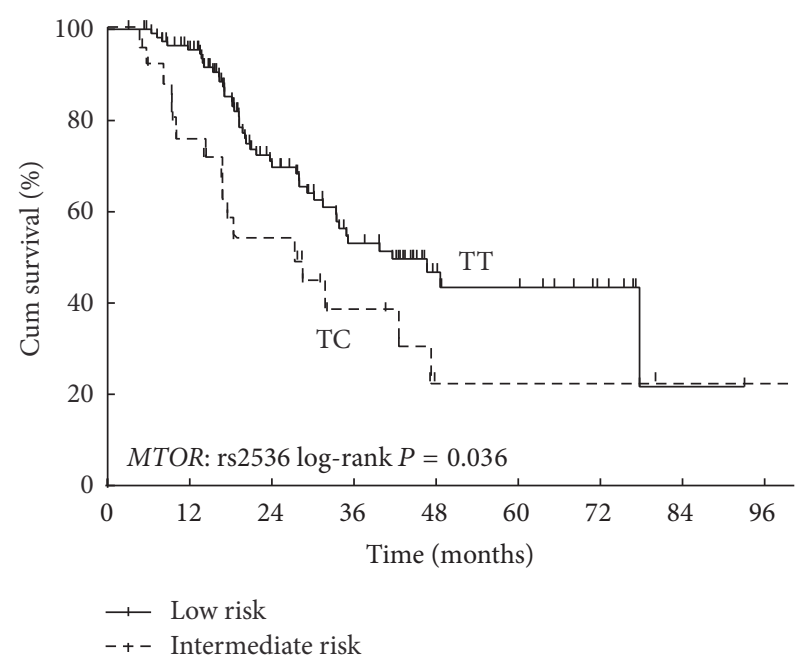

(b)

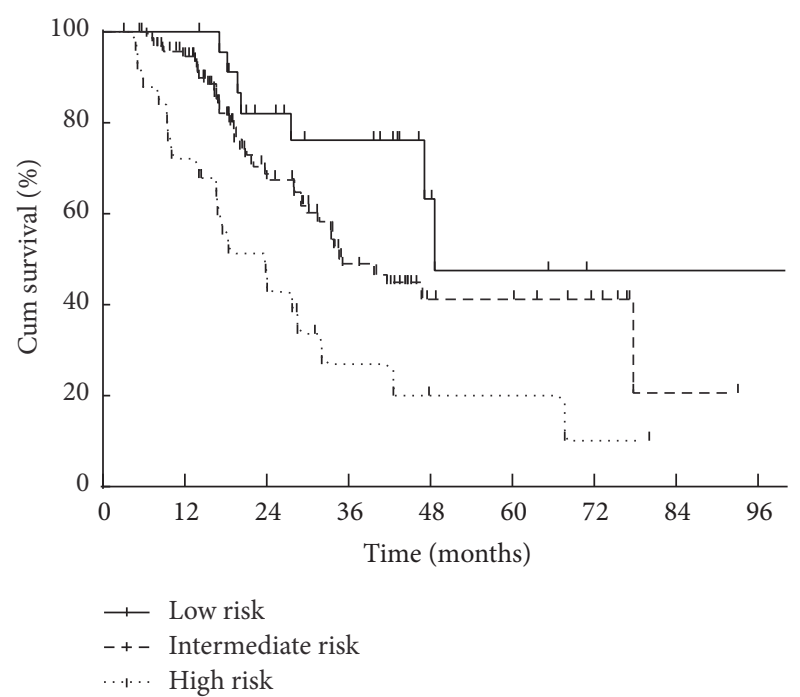

(d)

Figure 1: The Kaplan-Meier survival curve of selected SNPs and the RPA classification in patients with limited-disease small cell lung cancer treated with curative chemoradiotherapy. (a) PIK3R1: rs12755 (A>C); (b) MTOR: rs2536 (T>C); (c) PIK3R1: rs3756668 (A>G); (d) RPA classification.

the development of resistance to radiation and chemotherapy [20-22]. Genetic variants in this pathway were demonstrated to be associated with platinum-based chemotherapy response and prognosis in patients with advanced NSCLC [23]. In this study, we performed a pathway-specific analysis to determine whether polymorphisms in the PI3K/Akt/mTOR pathway may predict outcomes of patients with LD SCLC treated with curative chemoradiotherapy. Three SNPs in miRNA-binding genes in this pathway were identified to be associated with overall survival of these patients, and the unfavorable genotypes of MTOR: rs2536 (T>C) and PIK3R1: rs3756668 $(A>G)$ were combined by RPA to optimize the prognostic value. To our knowledge, this is the first study to evaluate the pathway-based effect of polymorphisms in the $\mathrm{PI} 3 \mathrm{~K} / \mathrm{Akt} / \mathrm{mTOR}$ pathway on clinical outcome of SCLC. The combination of multigenic variants classified these patients into three risk groups and provided a basis to identify patients with LD SCLC that will benefit from chemoradiotherapy.

The MTOR gene, located in exon 59 of chromosome 1p36.2, encodes a serine/threonine kinase and emerges as a key downstream effector of the PI3K/Akt/mTOR signaling pathway. Moreover, MTOR has been identified as a potential target in SCLC [22]. rs2536 is the mostly frequently studied polymorphism to be associated with cancer susceptibility in the $3^{\prime}$ UTR of this gene. However, reports of the association vary in the literature. MTOR rs2536 TC/CC genotypes were reported to have an association with decreased risk in acute lymphoblastic leukemia [24], but increased risk in prostate cancer [25], and no association in gastric and esophageal cancer $[26,27]$. Therefore, it is possible that the effect of this polymorphism on MTOR expression may be cancer type-specific. Until now, there has been no 


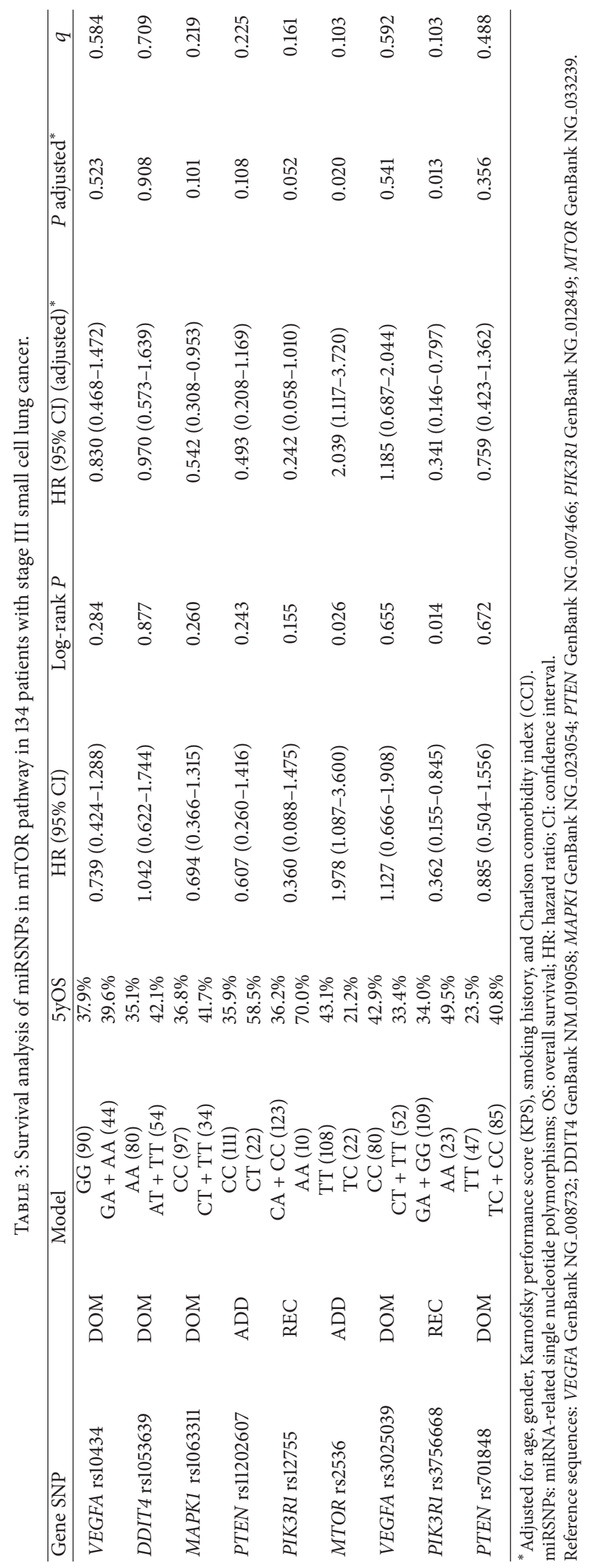




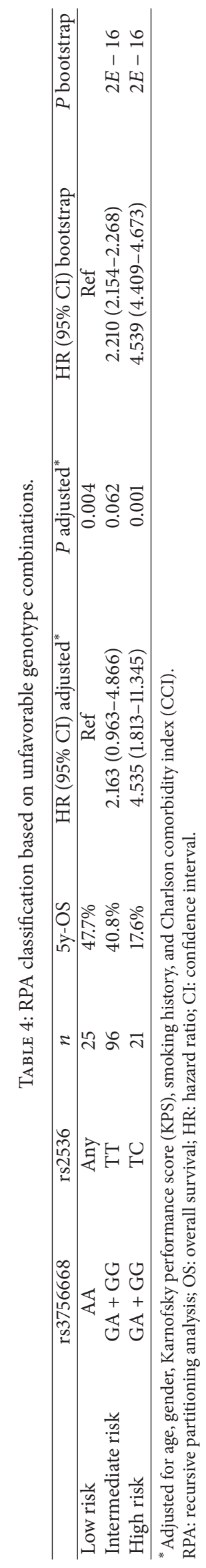


published work on the association between MTOR: rs2536 and cancer outcomes. In the present study, patients with rs2536 TC genotype showed a poorer survival than those with a TT genotype. As proposed previously, the binding miRNA-576 at the $\mathrm{T}$ allele was substituted for miRNA767 at the $\mathrm{C}$ allele predicted by the bioinformatics web server (https://snpinfo.niehs.nih.gov/snpinfo/snpfunc.php) [25]. Therefore, rs2536 TC genotype was speculated to affect MTOR expression, probably because of its effect on affinity for miRNA; however, MTOR expression is very complicated. It was found that phosphorylated MTOR ( $p$-MTOR) was more highly expressed in limited-stage than extended-stage SCLCs, and higher $p$-MTOR expression was associated with better prognosis [28], in contrast to other cancers [29, 30]. Therefore, elucidation of the exact mechanisms requires additional functional studies.

Two SNPs of PIK3R1, rs12755 (A>C) and rs3756668 $(A>G)$, were also found to be associated with survival in SCLC. Class IA PI3K, composed of a p110 catalytic unit and a regulatory unit, is activated by growth factors and subsequently induces a kinase cascade downstream [31]. PIK3R1 encodes the $85-\mathrm{kD}$ regulatory subunit, which is indispensable for stabilization of the catalytic subunit and downregulating PI3K signaling [32]. A handful of studies showed that polymorphisms of PIK3R1 were associated with survival in bladder cancer and endometrial cancer $[33,34]$. Neither PIK3R1 SNP discussed in this study has been reported to date. These PIK3R1 SNPs were found to be associated with favorable survival under a recessive model. Moreover, Ueki et al. [35] demonstrated that heterozygous disruption of PIK3R 1 reduced $\mathrm{p} 85 \alpha$ by $50 \%$ and enhanced PI3K signaling, while complete depletion of $\mathrm{p} 85 \alpha$ in homozygous generated knockout cells significantly decreased PI3-kinase activity. This may be indicative of the functional effect of these loci on PIK3R1 regulation and activity.

mTOR inhibitors displayed limited antitumor activity in SCLC on account of feedback reactivation of this pathway [36]. Dual inhibition of PI3K and mTOR can block the loop and enhance therapeutic effectiveness [18], and inhibition has been shown to improve tumor radiosensitivity by impairing DNA damage repair and normalizing tumor vasculature [37, 38]. Tumor activity depends on complex signaling networks. Thus, recent studies of cancer-related genetic alternations have also taken a pathway-based approach. In the present work, the combination of two SNPs in PIK3R1 and MTOR provided a more precise discrimination than individual SNPs. Consequently, it is biologically plausible for these potentially functional polymorphisms to have a synergistic effect.

To summarize, this study found three miRNA-related SNPs (MTOR: rs2536 (T>C), PIK3R1: rs3756668 (A>C) and PIK3R1: $r$ 12755 $(\mathrm{A}>\mathrm{G}))$ in the PI3K/Akt/mTOR pathway and pathway-based RPA that were associated with survival in patients with LD SCLC treated with chemoradiotherapy. The identification of these predictive factors may be help in selecting the optimal therapeutic strategy for individual patients. However, only nine functional SNPs were selected, based on prior knowledge, for the present study, which means that some important variations in this pathway may have been missed. In addition, multiple correction for these SNPs by 1000 bootstrap samples showed significant associations with OS, suggesting that the negative results after multiple correction were due to the limited number of subjects, although this is the largest study on miRNA-SNPs associated with prognosis in SCLC. Moreover, despite internal validation by bootstrap, there is a lack of independent external validation to confirm our findings. Therefore, additional larger, well-designed studies, including molecular mechanism investigation, are imperative to confirm these findings.

\section{Conclusions}

In conclusion, the current study identified 3 miRNArelated SNPs in the PI3K/Akt/mTOR pathway as prognostic biomarkers for patients with limited-disease SCLC treated with chemoradiotherapy. A risk classification incorporating MTOR: rs2536 (T>C) and PIK3R1: rs3756668 (A>C) was developed to identify patients likely to benefit from treatment. Replication in a large independent cohort and assessment of biological function is imperative to confirm these findings.

\section{Competing Interests}

The authors declare that there is no conflict of interests regarding the publication of this paper.

\section{Acknowledgments}

This work was supported by the National Nature Science Foundation of China (no. 81572971). The advice of Professor Xianming Tan of the Department of Biostatistics and the Lineberger Comprehensive Cancer Center at the University of North Carolina at Chapel Hill in statistical analysis is gratefully acknowledged.

\section{References}

[1] R. Govindan, N. Page, D. Morgensztern et al., "Changing epidemiology of small-cell lung cancer in the United States over the last 30 years: analysis of the surveillance, epidemiologic, and end results database," Journal of Clinical Oncology, vol. 24, no. 28, pp. 4539-4544, 2006.

[2] S. Sundstrøm, R. M. Bremnes, S. Kaasa et al., "Cisplatin and etoposide regimen is superior to cyclophosphamide, epirubicin, and vincristine regimen in small-cell lung cancer: results from a randomized phase III trial with 5 years' follow-up," Journal of Clinical Oncology, vol. 20, no. 24, pp. 4665-4672, 2002.

[3] M. Sorensen, U. Lassen, T. Palshof et al., "Topotecan and cisplatin in combination with concurrent twice-daily chemoradiation in limited disease small cell lung cancer-a Danish Oncological Lung Cancer Group (DOLG) phase II trial," Lung Cancer, vol. 60, no. 2, pp. 252-258, 2008.

[4] A. T. Turrisi III, K. Kim, R. Blum et al., “Twice-daily compared with once-daily thoracic radiotherapy in limited small-cell lung cancer treated concurrently with cisplatin and etoposide," The New England Journal of Medicine, vol. 340, no. 4, pp. 265-271, 1999.

[5] M. Marinov, A. Ziogas, O. E. Pardo et al., "AKT/mTOR pathway activation and BCL-2 family proteins modulate the sensitivity of 
human small cell lung cancer cells to RAD001," Clinical Cancer Research, vol. 15, no. 4, pp. 1277-1287, 2009.

[6] O. E. Pardo, A. Arcaro, G. Salerno et al., "Novel cross talk between MEK and S6K2 in FGF-2 induced proliferation of SCLC cells," Oncogene, vol. 20, no. 52, pp. 7658-7667, 2001.

[7] E. T. Shinohara and A. Maity, "Increasing sensitivity to radiotherapy and chemotherapy by using novel biological agents that alter the tumor microenvironment," Current Molecular Medicine, vol. 9, no. 9, pp. 1034-1045, 2009.

[8] X. Pu, M. A. T. Hildebrandt, C. Lu et al., "PI3K/PTEN/ $\mathrm{AKT} / \mathrm{mTOR}$ pathway genetic variation predicts toxicity and distant progression in lung cancer patients receiving platinumbased chemotherapy," Lung Cancer, vol. 71, no. 1, pp. 82-88, 2011.

[9] M. A. T. Hildebrandt, H. Yang, M.-C. Hung et al., "Genetic variations in the PI3K/PTEN/AKT/mTOR pathway are associated with clinical outcomes in esophageal cancer patients treated with chemoradiotherapy," Journal of Clinical Oncology, vol. 27, no. 6, pp. 857-871, 2009.

[10] G. C. Shukla, J. Singh, and S. Barik, "MicroRNAs: processing, maturation, target recognition and regulatory functions," Molecular and Cellular Pharmacology, vol. 3, no. 3, pp. 83-92, 2011.

[11] J. A. McCubrey, L. S. Steelman, C. R. Kempf et al., "Therapeutic resistance resulting from mutations in Raf/MEK/ERK and PI3K/PTEN/Akt/mTOR signaling pathways," Journal of Cellular Physiology, vol. 226, no. 11, pp. 2762-2781, 2011.

[12] P. Micke, A. Faldum, T. Metz et al., "Staging small cell lung cancer: veterans administration lung study group versus international association for the study of lung cancer-what limits limited disease?” Lung Cancer, vol. 37, no. 3, pp. 271-276, 2002.

[13] T. B. Rounge, M. Lauritzen, H. Langseth, E. Enerly, R. Lyle, and R. E. Gislefoss, "MicroRNA biomarker discovery and highthroughput DNA sequencing are possible using long-term archived serum samples," Cancer Epidemiology Biomarkers \& Prevention, vol. 24, no. 9, pp. 1381-1387, 2015.

[14] M. Gonen and G. Heller, "Concordance probability and discriminatory power in proportional hazards regression," Biometrika, vol. 92, no. 4, pp. 965-970, 2005.

[15] Y. Benjamini and Y. Hochberg, "Controlling the false discovery rate-a practical and powerful approach to multiple testing," Journal of the Royal Statistical Society, Series B: Methodological, vol. 57, no. 1, pp. 289-300, 1995.

[16] M. Peifer, L. Fernandez-Cuesta, M. L. Sos et al., "Integrative genome analyses identify key somatic driver mutations of smallcell lung cancer," Nature Genetics, vol. 44, no. 10, pp. 1104-1110, 2012.

[17] M. S. Lawrence, P. Stojanov, P. Polak et al., "Mutational heterogeneity in cancer and the search for new cancer-associated genes," Nature, vol. 499, no. 7457, pp. 214-218, 2013.

[18] S. Umemura, S. Mimaki, H. Makinoshima et al., "Therapeutic priority of the PI3K/AKT/mTOR pathway in small cell lung cancers as revealed by a comprehensive genomic analysis," Journal of Thoracic Oncology, vol. 9, no. 9, pp. 1324-1331, 2014.

[19] H. Cheng, M. Shcherba, G. Pendurti, Y. Liang, B. Piperdi, and R. Perez-Soler, "Targeting the PI3K/AKT/mTOR pathway: potential for lung cancer treatment," Lung Cancer Management, vol. 3, no. 1, pp. 67-75, 2014.

[20] J. Bussink, A. J. van der Kogel, and J. H. Kaanders, "Activation of the PI3-K/AKT pathway and implications for radioresistance mechanisms in head and neck cancer," The Lancet Oncology, vol. 9, no. 3, pp. 288-296, 2008.
[21] L.-Z. Liu, X.-D. Zhou, G. Qian, X. Shi, J. Fang, and B.$\mathrm{H}$. Jiang, "AKT1 amplification regulates cisplatin resistance in human lung cancer cells through the mammalian target of rapamycin/p70s6K1 pathway," Cancer Research, vol. 67, no. 13, pp. 6325-6332, 2007.

[22] G. W. Krystal, G. Sulanke, and J. Litz, "Inhibition of phosphatidylinositol 3-kinase-akt signaling blocks growth, promotes apoptosis, and enhances sensitivity of small cell lung cancer cells to chemotherapy," Molecular Cancer Therapeutics, vol. 1, no. 11, pp. 913-922, 2002.

[23] J.-L. Xu, Z.-W. Wang, L.-M. Hu et al., "Genetic variants in the PI3K/PTEN/AKT/mTOR pathway predict platinum-based chemotherapy response of advanced non-small cell lung cancers in a Chinese population," Asian Pacific Journal of Cancer Prevention, vol. 13, no. 5, pp. 2157-2162, 2012.

[24] L. Huang, J. Huang, P. Wu et al., "Association of genetic variations in mTOR with risk of childhood acute lymphoblastic leukemia in a Chinese population," Leukemia and Lymphoma, vol. 53, no. 5, pp. 947-951, 2012.

[25] Q. Li, C. Gu, Y. Zhu et al., "Polymorphisms in the mTOR gene and risk of sporadic prostate cancer in an Eastern Chinese population," PLoS ONE, vol. 8, no. 8, Article ID e71968, 2013.

[26] J. He, M.-Y. Wang, L.-X. Qiu et al., "Genetic variations of mTORC1 genes and risk of gastric cancer in an Eastern Chinese population," Molecular Carcinogenesis, vol. 52, supplement 1, pp. E70-E79, 2013.

[27] M.-L. Zhu, H. Yu, T.-Y. Shi et al., "Polymorphisms in mTORC1 genes modulate risk of esophageal squamous cell carcinoma in eastern Chinese populations," Journal of Thoracic Oncology, vol. 8, no. 6, pp. 788-795, 2013.

[28] J. H. Lee, K. W. Kang, and H. W. Lee, "Expression of phosphorylated mTOR and its clinical significances in small cell lung cancer," International Journal of Clinical and Experimental Pathology, vol. 8, no. 3, pp. 2987-2993, 2015.

[29] J. Y. An, K. M. Kim, M. G. Choi et al., "Prognostic role of pmTOR expression in cancer tissues and metastatic lymph nodes in p T2b gastric cancer," International Journal of Cancer, vol. 126, no. 12, pp. 2904-2913, 2010.

[30] T. Dhillon, F. A. Mauri, G. Bellezza et al., "Overexpression of the mammalian target of rapamycin: a novel biomarker for poor survival in resected early stage non-small cell lung cancer," Journal of Thoracic Oncology, vol. 5, no. 3, pp. 314-319, 2010.

[31] J. A. Engelman, J. Luo, and L. C. Cantley, "The evolution of phosphatidylinositol 3-kinases as regulators of growth and metabolism," Nature Reviews Genetics, vol. 7, no. 8, pp. 606-619, 2006.

[32] J. Luo and L. C. Cantley, "The negative regulation of phosphoinositide 3-kinase signaling by $\mathrm{p} 85$ and it's implication in cancer," Cell Cycle, vol. 4, no. 10, pp. 1309-1312, 2005.

[33] L.-E. Wang, H. Ma, K. S. Hale et al., "Roles of genetic variants in the PI3K and RAS/RAF pathways in susceptibility to endometrial cancer and clinical outcomes," Journal of Cancer Research and Clinical Oncology, vol. 138, no. 3, pp. 377-385, 2012.

[34] M. Chen, J. Gu, G. L. Delclos et al., "Genetic variations of the PI3K-AKT-mTOR pathway and clinical outcome in muscle invasive and metastatic bladder cancer patients," Carcinogenesis, vol. 31, no. 8, pp. 1387-1391, 2010.

[35] K. Ueki, D. A. Fruman, S. M. Brachmann, Y.-H. Tseng, L. C. Cantley, and R. C. Kahn, "Molecular balance between the regulatory and catalytic subunits of phosphoinositide 3-kinase regulates cell signaling and survival," Molecular and Cellular Biology, vol. 22, no. 3, pp. 965-977, 2002. 
[36] S.-Y. Sun, L. M. Rosenberg, X. Wang et al., "Activation of Akt and eIF4E survival pathways by rapamycin-mediated mammalian target of rapamycin inhibition," Cancer Research, vol. 65, no. 16, pp. 7052-7058, 2005.

[37] C. R. Gil Del Alcazar, M. C. Hardebeck, B. Mukherjee et al., "Inhibition of DNA double-strand break repair by the dual PI3K/mTOR inhibitor NVP-BEZ235 as a strategy for radiosensitization of glioblastoma," Clinical Cancer Research, vol. 20, no. 5, pp. 1235-1248, 2014.

[38] E. Fokas, J. H. Im, S. Hill et al., "Dual inhibition of the $\mathrm{PI} 3 \mathrm{~K} / \mathrm{mTOR}$ pathway increases tumor radiosensitivity by normalizing tumor vasculature," Cancer Research, vol. 72, no. 1, pp. 239-248, 2012. 


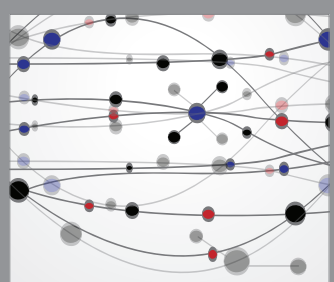

The Scientific World Journal
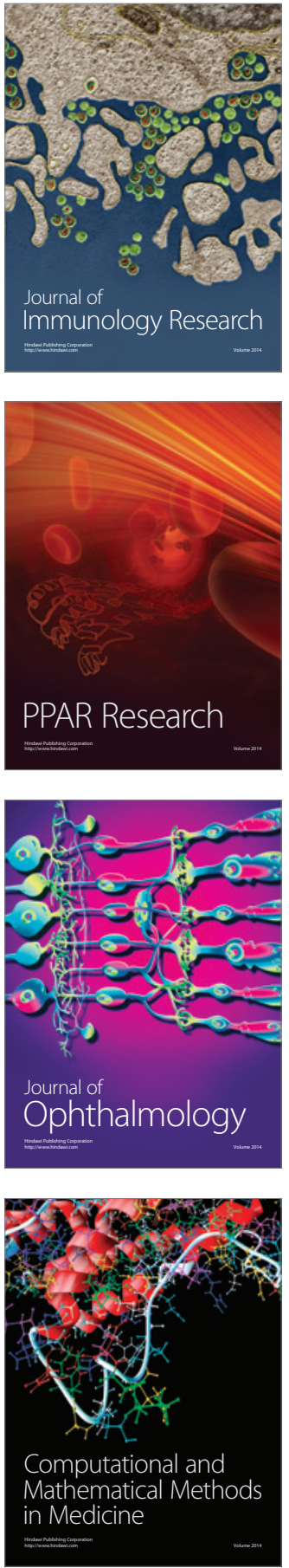

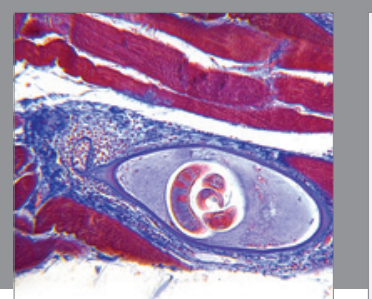

Gastroenterology Research and Practice
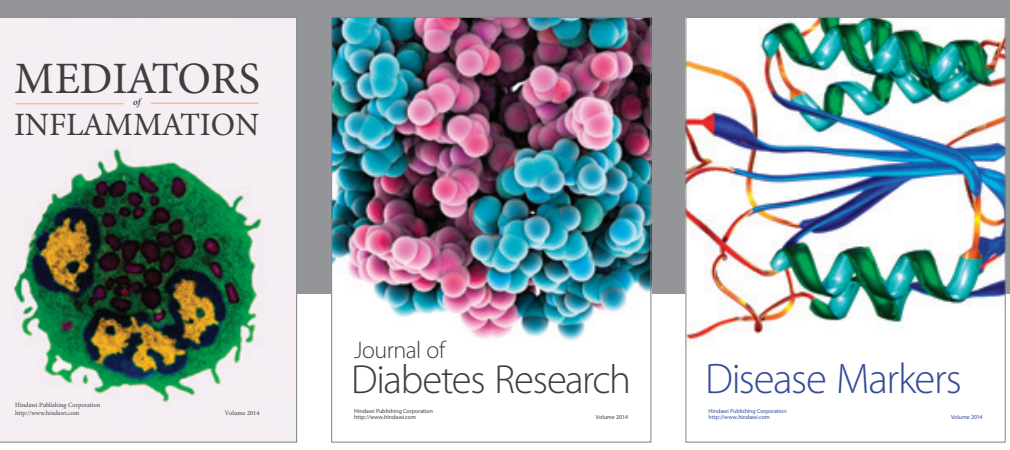

Disease Markers

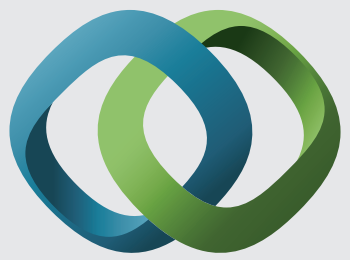

\section{Hindawi}

Submit your manuscripts at

https://www.hindawi.com
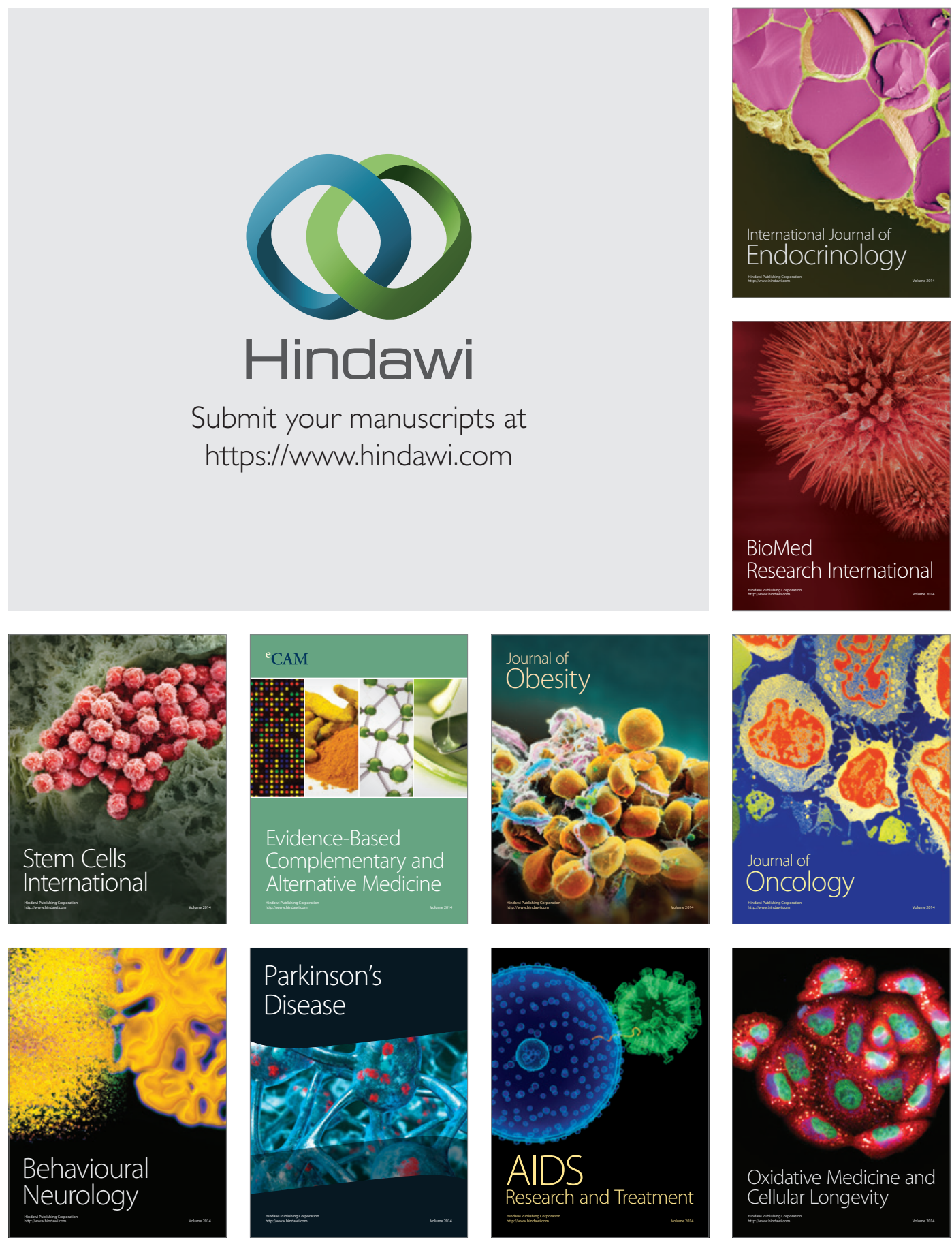